ARTICLE

\title{
Spectral phase transitions in optical parametric oscillators
}

\author{
Arkadev Roy ${ }^{1}$, Saman Jahani (iD ${ }^{1}$, Carsten Langrock ${ }^{2}$, Martin Fejer $^{2}$ \& Alireza Marandi (i) ${ }^{1 凶}$
}

Driven nonlinear resonators provide a fertile ground for phenomena related to phase transitions far from equilibrium, which can open opportunities unattainable in their linear counterparts. Here, we show that optical parametric oscillators (OPOs) can undergo second-order phase transitions in the spectral domain between degenerate and non-degenerate regimes. This abrupt change in the spectral response follows a square-root dependence around the critical point, exhibiting high sensitivity to parameter variation akin to systems around an exceptional point. We experimentally demonstrate such a phase transition in a quadratic OPO. We show that the divergent susceptibility of the critical point is accompanied by spontaneous symmetry breaking and distinct phase noise properties in the two regimes, indicating the importance of a beyond nonlinear bifurcation interpretation. We also predict the occurrence of first-order spectral phase transitions in coupled OPOs. Our results on nonequilibrium spectral behaviors can be utilized for enhanced sensing, advanced computing, and quantum information processing.

\footnotetext{
${ }^{1}$ Department of Electrical Engineering, California Institute of Technology, Pasadena, CA 91125, USA. ${ }^{2}$ Edward L. Ginzton Laboratory, Stanford University, Stanford, CA 94305, USA. ${ }^{\circledR}$ email: marandi@caltech.edu
} 
P hotonic resonators appearing in myriad forms ranging from macro-scale to nano-scale have been the mainstay of lightbased fundamental studies and applications ${ }^{1}$. The ability to engineer the resonant spectral features of these cavities unveil tremendous possibilities in sensing and light-matter interactions. The interplay of gain/loss and coupling in coupled linear photonic resonators can lead to the occurrence of a multitude of intriguing phenomena ranging from Fano resonance, electromagnetically induced transparency, ${ }^{2}$ and exceptional point associated with parity-time symmetry breaking 3,4 .

Strong nonlinearities in photonic resonators can lead to a variety of rich phenomena. Nonlinear driven dissipative systems existing in non-equilibrium steady states exhibit selforganization $^{5}$, pattern formation ${ }^{6-10}$, and emergent phase and dynamical phase transitions ${ }^{11}$. Other salient examples include behaviors in laser systems $s^{12-14}$ at threshold ${ }^{15}$ and around modelocking transitions ${ }^{16-18}$, soliton-steps in Kerr microresonators ${ }^{19}$, and in polaritonic quantum fluids ${ }^{20}$. Similar phenomena are also explored outside photonics for instance in the form of Rayleigh-Benard convection and Faraday waves ${ }^{21,22}$. Specific to the parametric oscillation regime, a variety of nonlinear dynamical behaviors has been predicted and demonstrated, such as bistability, self-pulsation, limit-cycles ${ }^{23}$, pattern formation ${ }^{6-10}$ and phase transitions ${ }^{24,25}$, albeit not explicitly in the spectral domain.

Phase transition marks a universal qualitative regime change in system properties as the control parameter is varied around a critical/transition point ${ }^{26}$. The behavior of the system around the critical point is characterized by the order parameter (OP). Second-order phase transition displays continuity in the OP while exhibiting a discontinuity in the derivative of the OP. On the other hand, first-order transition is known to possess a discontinuous OP around the transition point.

Realizing phase transitions based on the optical parametric processes can provide unique opportunities for sensing. For instance, in phase transition-based detectors and transitionedge sensors ${ }^{27}$, the reset time (return time to the critical bias point) can be significantly reduced using an ultrafast nonlinear process compared to thermodynamic transitions. Moreover, similar to the exceptional points in optical systems ${ }^{3,4}$, an enhanced sensitivity 28,29 can be realized using a driven dissipative-based phase transition. However, in contrast to exceptional points in PT-symmetric systems, this enhancement is not accompanied by eigenvectors non-orthogonality and can potentially provide high sensitivity combined with high precision $^{30,31}$. The noiseless nature of parametric amplification $^{32}$ can be another unique resource for enhancing the signal-to-noise ratio; a property that is not readily available in current implementations of exceptional points. Divergent susceptibility of the critical point supported by the parametric gain in a driven-dissipative setting can open unexplored avenues in the context of non-Hermitian sensing.

In this work, we exploit the rich dynamics of nonlinear driven dissipative systems in OPOs to formulate and engineer their spectral behaviors as phase transitions. We demonstrate second-order spectral phase transition in quadratic OPOs and identify the critical point demarcating the degenerate and non-degenerate oscillation regimes and the associated spontaneous symmetry breaking. We map the OPO dynamics to the universal Swift-Hohenberg equation ${ }^{33,34}$ and extend it to Kerr OPOs. We predict the occurrence of complex collective behavior like first-order phase transitions in coupled OPOs. Our results on non-equilibrium spectral behaviors can be utilized for enhanced sensing that leverages the diverging susceptibility of the critical point, and advanced computing paradigms exploiting the criticality of the spectral phase transition.

\section{Results}

We consider a doubly resonant $\mathrm{OPO}^{35,36}$ as a driven-dissipative system in a non-equilibrium steady state. The OPO driving is accomplished by the synchronous pulsed pump centered around the frequency $2 \omega_{0}$, while the resonant signal and the idler constitute the longitudinal modes of the resonator centered around the half-harmonic frequency $\left(\omega_{0}\right)$. The interaction among the modes is engendered by the quadratic nonlinearity (Fig. 1a). The inherent coupled nature of the signal and idler in a doubly resonant OPO gives rise to rich nonlinear dynamics including the appearance of bi-phase states around degeneracy ${ }^{37}$. The mutual interplay between the cavity detuning and the temporal groupvelocity dispersion (GVD) provides another degree of freedom, which governs the dynamics of signal/idler in synchronously pumped doubly resonant OPOs. This leads to discontinuities typical of a second-order phase transition around the critical cavity detuning (Fig. 1b, c). This spectral phase transition demarcates a sharp boundary between the degenerate and nondegenerate parametric oscillation.

In the Continuous Wave (CW)-driven high-finesse limit, the $\mathrm{OPO}$ is governed by the mean-field evolution equation:

$$
\begin{aligned}
\frac{\partial a}{\partial \xi}= & (-\alpha+i \Delta \phi) a+g a^{*}-i \frac{\beta_{2}}{2} \frac{\partial^{2} a}{\partial t^{2}} \\
& -\left[\frac{\epsilon^{2}}{2 u^{2}} \int_{0}^{L u}(L u-\tau) a(t-\tau)^{2} d \tau\right] a^{*},
\end{aligned}
$$

where, $a$ describes the signal envelope under the slowly varying envelope approximation limit. Here $\xi, t$ refers to the slow time and the fast time, respectively ${ }^{38} . \alpha, \Delta \phi, \beta_{2}$, and $g$ denote the loss, detuning, GVD, and the phase-sensitive parametric gain, respectively. $g$ in the $\mathrm{CW}$-limit is expressed as $\epsilon b L$, where $b$ is the pump amplitude. $L$ refers to the cavity round trip length where the nonlinear interaction is encountered, $\epsilon$ includes the strength of the nonlinear interaction and $u$ is the walk-off parameter. The last term on the right hand side of the equation is responsible for the gain saturation. Each of these terms is normalized by suitable normalization factors (see Supplementary Information, Section 3).

We assume a perturbation in the field (signal/idler) around the half-harmonic frequency $\left(\omega_{0}\right)$ to be of the form: $a=a_{+} e^{i \delta \omega t}+$ $a_{-} e^{-i \delta \omega t}$. We perform linear stability analysis (neglecting gain saturation) to determine the most unstable longitudinal mode, which is given as:

$$
\begin{gathered}
\frac{d}{d \xi}\left[\begin{array}{l}
a_{+} \\
a_{-}^{*}
\end{array}\right]=\left[\begin{array}{cc}
-\alpha+i \Gamma & g \\
g^{*} & -\alpha-i \Gamma
\end{array}\right]\left[\begin{array}{l}
a_{+} \\
a_{-}^{*}
\end{array}\right] \\
\lambda_{ \pm}=-\alpha \pm \sqrt{|g|^{2}-\Gamma^{2}}
\end{gathered}
$$

where $\Gamma=\Delta \phi+\frac{\beta_{2}}{2}(\delta \omega)^{2}$. Analyzing the eigenvalue (growth rate) (Eq. (2b)) of the linear stability matrix we arrive at two scenarios. First, when $\operatorname{sgn}(\Delta \phi)=\operatorname{sgn}\left(\beta_{2}\right)$, we find that the most unstable frequency of oscillation is $\delta \omega=0$, and the corresponding threshold (i.e., when $\lambda_{+}=0$ ) is $|g|_{\text {th }}=\sqrt{\alpha^{2}+(\Delta \phi)^{2}}$, leaving the OPO in the degenerate phase. However, when $\operatorname{sgn}(\Delta \phi)=-\operatorname{sgn}\left(\beta_{2}\right)$, the most unstable frequency of oscillation is given by $\delta \omega=\left|\frac{2 \Delta \phi}{\beta_{2}}\right|^{\frac{1}{2}}$, and the associated threshold is $|g|_{\text {th }}=\alpha$, leaving the OPO in the non-degenerate phase. This can be understood as cavity detuning $(\Delta \phi)$ counterbalancing the GVD induced detuning in the non-degenerate regime. This can happen for positive cavity detuning in the anomalous regime, where GVD induced detuning is negative and they cancel exactly at $\omega_{0} \pm \delta \omega$, thereby experiencing higher gain in the non-degenerate phase resulting in OPO selecting non-degeneracy over degenerate oscillation. This proves the existence of the spectral phase transition which is 


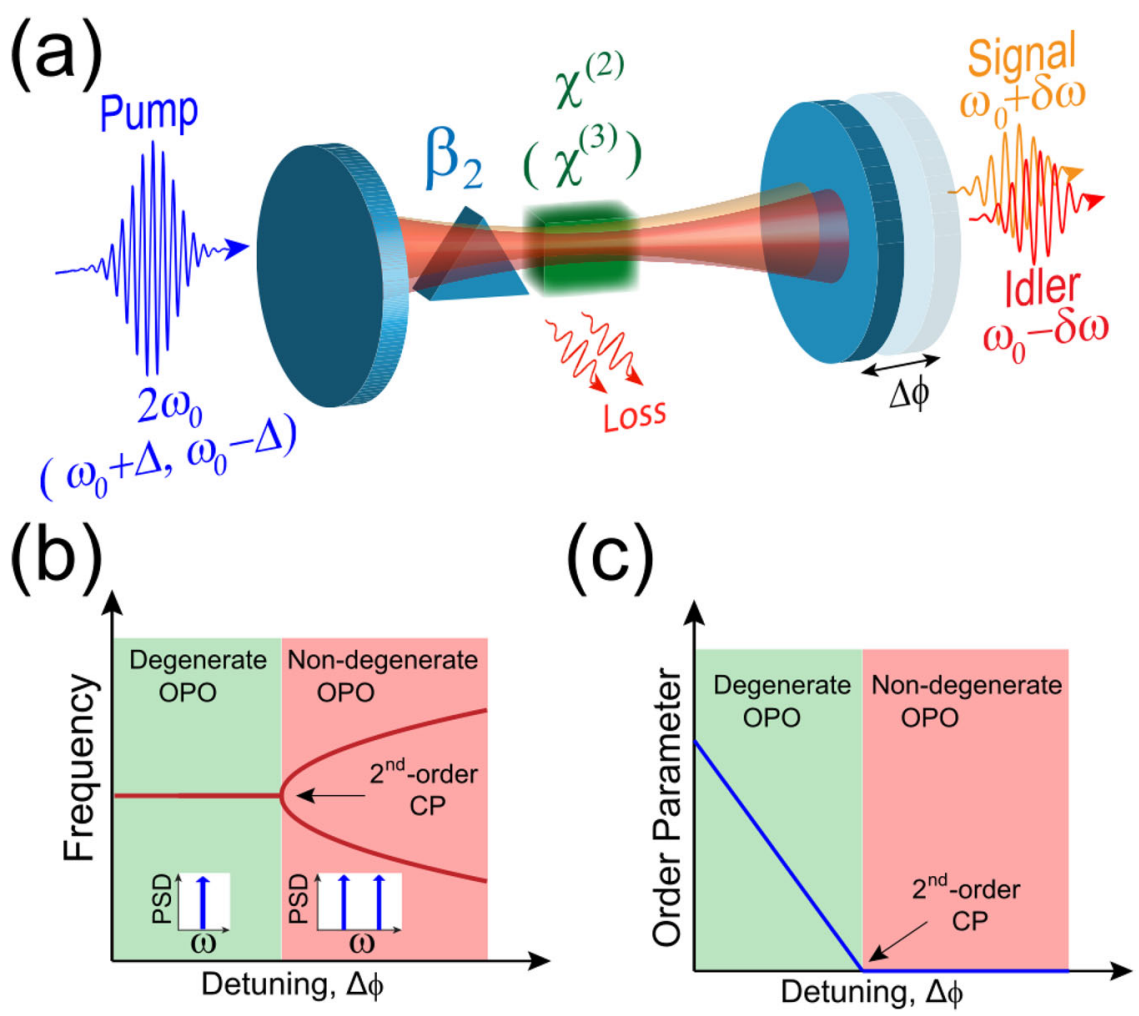

Fig. 1 Spectral phase transition in nonlinear photonic resonators. a OPO with the resonant signal and idler in the cavity with variable detuning $(\Delta \phi)$ and second-order group-velocity dispersion $\left(\beta_{2}\right)$. The nonlinearity can be provided by a quadratic $\left(\chi^{(2)}\right)$ or a Kerr $\left(\chi^{(3)}\right)$ medium. b A second-order phase transition occurs at the critical detuning that marks the transition between the degenerate and the non-degenerate spectrum. $\mathbf{c}$ This transition is characterized by a continuous order parameter, but a discontinuous derivative of the order parameter at the critical point (CP).

demonstrated in Fig. 2. The spectral phase transition takes place around the detuning, $\Delta \phi=0$. The behavior in the normal GVD regime (Fig. 2d) is reversed as compared to the anomalous GVD scenario (Fig. 2b). Results obtained experimentally (Fig. 2c, e) agree well with the simulations.

The spectral transition can be interpreted as an order-disorder transition whereby the OPO transits from an ordered bi-phase state in the degenerate regime, to a disordered phase state in the non-degenerate regime with the signal assuming random phases and the idler following it (see Supplementary Information, Section 15). Thus the critical point marks the onset of the spontaneous $U(1)$ symmetry breaking. In our context, we define OP as, $O P=\frac{d \lambda_{\max }}{d \Delta \phi}$, which represents the derivative of the gain with respect to the detuning. The gain $\left(\lambda_{\max }=\max _{\delta \omega} \lambda_{+}\right)$i.e., the maximum eigenvalue is obtained using Eq. (S8) (see Supplementary Information, Section 4). The phase-dependent parametric gain is sensitive to detuning induced phase accumulation more acutely in the degenerate regime as opposed to the nondegenerate regime where it varies slowly with detuning. The order-disorder transition has important implications in the phase noise and coherence properties of the OPO. While the phase noise of OPO operating at degeneracy is dominated by the driving pump, in the non-degenerate regime phase diffusion leads to the Schawlow-Townes limit for each of the signal and the idler $^{39}$, albeit with anti-correlation in their phases and potential phase-sum quadrature squeezing ${ }^{40}$. The phase transition description reveals interesting correlation properties in the disordered regime i.e., the non-degenerate regime. The phase difference diffusion follows a power-law dependence as a function of the detuning (i.e., distance from the critical point) which mimics the behavior of correlation functions in continuous phase transitions (see Supplementary Information, Section 9).
We further characterize the quadratic OPO around the phase transition point (Fig. 3). The critical point coincides with the maximum output power of the OPO as observed numerically and experimentally (Fig. 3a, b). This behavior can be explained by the gain calculations (inset of Fig. 3c). The threshold is a function of the detuning and the dispersion (see Supplementary Information, Section 4). The OP displays characteristics (Fig. 3c) typical of second-order phase transitions or soft transitions ${ }^{41}$. In addition, in the pulsed regime as the OPO undergoes the phase transition, the signal and idler combs split and interfere with each other with a beat frequency equal to the difference of their respective carrierenvelope offset frequencies. This leads to the spontaneous emergence of beat notes as shown in the measurement results of Fig. 3d. This is a manifestation of a critical slowing down phenomenon, where the time period of the beat-note tends to infinity as we approach the critical point from the non-degenerate regime. Note that, the detuning range of the parametric oscillation, as well as the ratio of degenerate and non-degenerate regimes above the threshold, is determined by the gain, which is a function of the pump power and the cavity dispersion (see Supplementary Information, Section 11).

When two OPOs are coupled, the transition from degenerate to non-degenerate operation can occur as a first-order phase transition. Figure $4 \mathrm{a}$ depicts a schematic representation of the coupled OPO configuration. In the presence of the coupling, the competition between the two second-order phase transitions (as shown by the gain curve in Fig. 4d) results in the emergence of a firstorder spectral phase transition (Fig. 4e). This first-order transition point causes a sudden discontinuity/hard transition in the spectrum (Fig. 4b, c) as the coupled OPO transits from the nondegenerate to the degenerate spectral regime (Fig. 4e). The coupling in the linear regime induces a mode splitting which is 


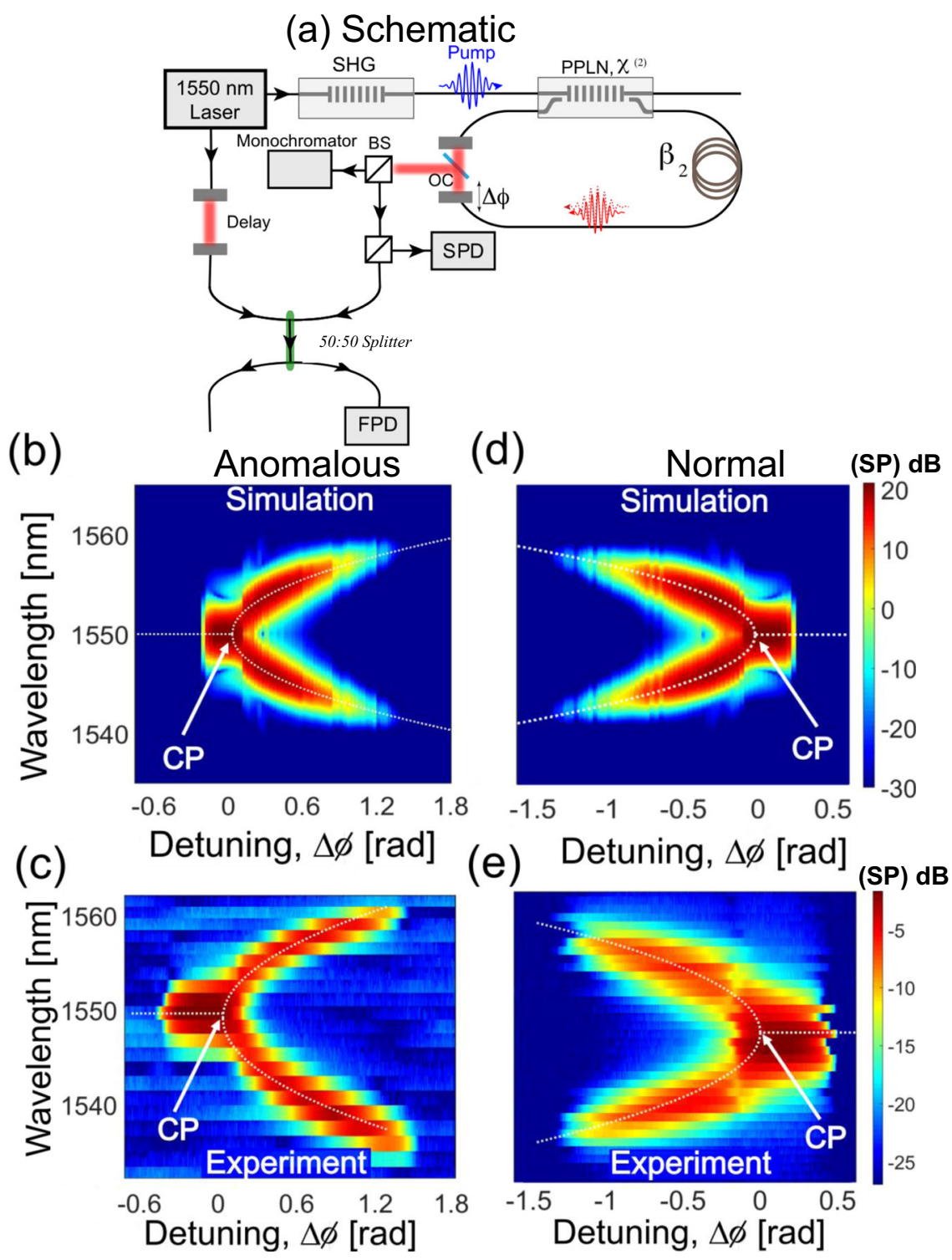

Fig. 2 Second-order spectral phase transition in an OPO. a Schematic of the experimental setup, which implements the spectral phase transition in a guided-wave OPO based on PPLN. BS beam splitter, OC output coupler, SPD slow photo-detector, FPD fast photo-detector, SHG second harmonic generation. Spectrum (SP) as a function of detuning obtained through numerical simulation $\mathbf{b}$ in anomalous dispersion regime (dotted line represents the theoretically expected spectral splitting, which in the non-degenerate regime is given by: $\delta \omega=\sqrt{\frac{-2 \Delta \phi}{\beta_{2}}}$, $\mathbf{d}$ in the normal dispersion regime. Experimental results capturing the second-order critical point $\mathbf{c}$ in anomalous dispersion regime, $\mathbf{e}$ in normal dispersion regime. It closely follows the square-root behavior (dotted line) in the non-degenerate regime. Color bar represents power spectral density in $\mathrm{dB}$.

expected to introduce a second-order phase transition around the split resonances as evident from the plot of OP in Fig. 4d. This can be understood by the argument that a positive cavity detuning applied to individual cavities can appear both as a positive or a negative detuning in the coupled basis depending on the relative magnitude of the cavity detuning and the coupling strength. Further details regarding the modeling of coupled OPOs are presented in the supplementary information (see Supplementary Information, Section 6, 7).

The demonstrated spectral phase transitions can be described by the universal Swift-Hohenberg equation which is also known to govern nonlinear pattern formation dynamics ${ }^{33,34}$. The mapping of the OPO dynamics to the Swift-Hohenberg equation is derived in S8 (see Supplementary Information, Section 8). The same equation we derived in this context can describe degenerate four-wave mixing dynamics contingent to certain conditions.
Thus, spectral phase transitions are also expected to occur in Kerr OPOs (Fig. 5a). For the Kerr OPO, we consider a conservative system governed by the nonlinear Schroedinger equation. $\frac{\partial A}{\partial z}=-i \frac{\beta_{2}}{2} \frac{\partial^{2} A}{\partial \tau^{2}}+i \gamma|A|^{2} A . \gamma$ represents the effective third-order nonlinear co-efficient and $\beta_{2}$ stands for the second-order GVD co-efficient. Degenerate parametric oscillation can be realized in a Kerr medium using dual pumps (Fig. 5b, c) ${ }^{42,43}$. We represent the dual pumps as having amplitudes $A_{1}$ and $A_{2}$ and assume that they have equal power $\left(P=\left|A_{1}\right|^{2}=\left|A_{2}\right|^{2}\right)$ and possesses a detuning of $\Delta$ from the center of degeneracy. Owing to the symmetry, we assume the parametrically generated signal $\left(A_{s}\right)$ and idler $\left(A_{i}\right)$ to be detuned by $\delta \omega$ from the center of degeneracy. We express the field envelope as given by the following expansion: $A(z, \tau)=$ $A_{1} e^{i \Delta \tau}+A_{2} e^{-i \Delta \tau}+A_{s}(z) e^{i \delta \omega \tau}+A_{i}(z) e^{-i \delta \omega \tau}$. Parametric gain at the onset of the phase conjugation parametric process can be determined via a linear stability analysis. The growth rate due to 
(a) Simulation

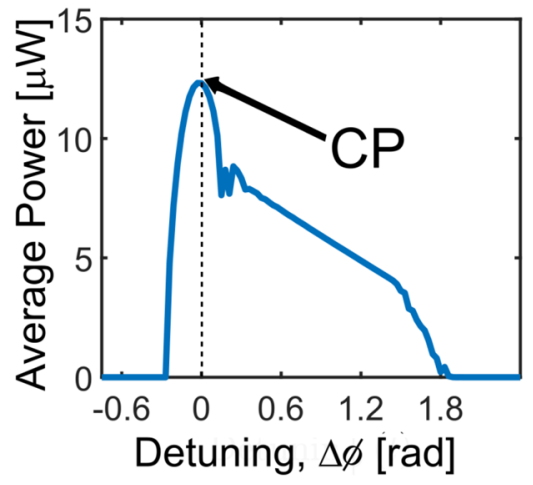

(c)

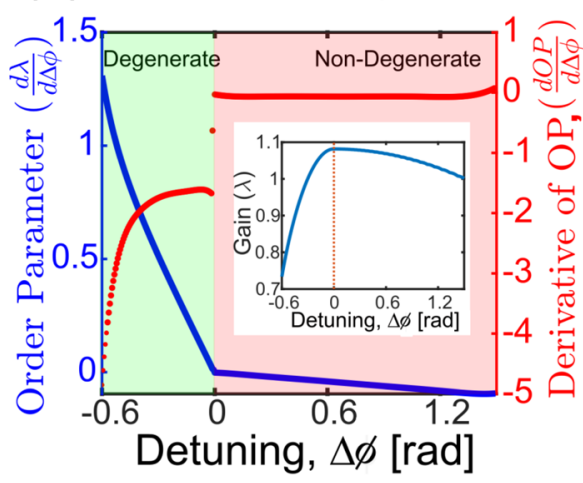

(b) Experiment

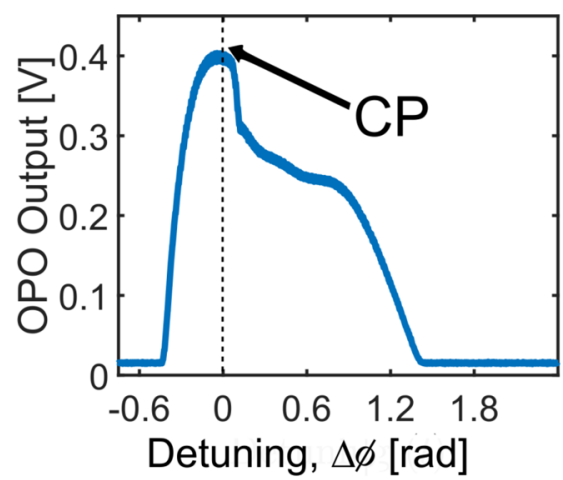

(d) Experiment

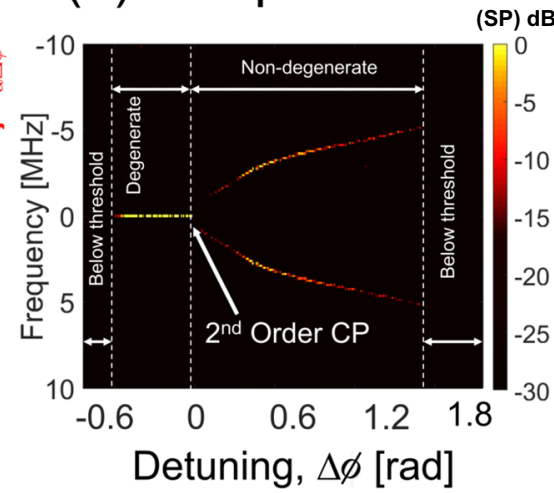

Fig. 3 Characterization of the second-order critical point. OPO average output power as a function of cavity detuning obtained numerically (a) and experimentally (b) using a slow detector. This demonstrates the maximum conversion efficiency at the critical point. c Continuous order parameter but discontinuous derivative typical of a second-order phase transition. The inset shows the gain curve as a function of cavity detuning, which has its maximum

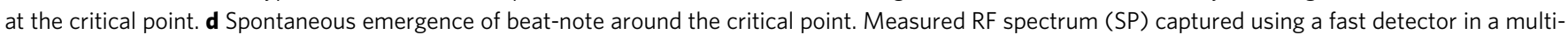
heterodyne measurement showing co-existence of the signal and idler combs in the non-degenerate regime and their offset tuning.

the phase conjugation parametric process can be expressed as $e^{\lambda z}$ where $\lambda$ is given by ${ }^{42}$ :

$$
\lambda=\sqrt{\left\{6 \gamma P-\beta_{2}\left(\Delta^{2}-(\delta \omega)^{2}\right)\right\}\left\{2 \gamma P+\beta_{2}\left(\Delta^{2}-(\delta \omega)^{2}\right)\right\}}
$$

The spectral phase transition is shown in Fig. $5 \mathrm{~d}$. The fact that the associated critical point is second-order is established by analyzing the OP as depicted in Fig. 5e.

\section{Discussion}

The abrupt frequency splitting around the critical point in these spectral phase transitions can be utilized for enhanced sensing. A sensor can be based on the second-order spectral phase transition biased at the critical point, that will exhibit a scaling of $\delta \omega \sim \varepsilon^{\frac{1}{2}}$, where $\varepsilon$ is a small perturbation (e.g., in detuning) under consideration, similar to a second-order exceptional point ${ }^{28}$. However, if we leverage the first-order spectral phase transition for a critical detector, we can utilize the discontinuity in the spectrum for highly enhanced sensitivity 27,44 . The proportionality constant in the scaling law is a function of the cavity GVD. The smaller the dispersion, higher is the sensitivity (see Supplementary Information, Section 5). Spectral phase transitions in OPOs can also open opportunities for computing and quantum information processing. Phase transition occurring at the oscillation threshold of OPOs has been utilized as a promising computing resource in optical Ising machines ${ }^{37,45}$. Phase transition occurring in the spectral domain can provide additional computing capabilities. For example, in the OPO-based Ising machines, which have been strictly operating at degeneracy so $\operatorname{far}^{37,45}$, the spectral phase transition can act as an additional search mechanism leveraging the symmetry breaking and additional phase noise in the non-degenerate regime. Moreover, our results on spectral phase transition can extend the foundation for phase transition-based computing platforms ${ }^{46}$.

Tuning the spectrum of parametric oscillation between degeneracy and non-degeneracy is a well-known concept, and the same is achieved by manipulating the phase-matching curve via temperature, voltage control, etc. ${ }^{47}$. Distinctively, the presented spectral phase transition occurs as a multimode co-operative phenomenon ${ }^{14}$ triggered by cavity phase detuning, where dispersion plays a crucial role, while the phase matching enabled by the periodically poled waveguide remains unaltered.

The presented spectral phase transition is in sharp contrast to intensity-dependent bifurcation ubiquitous in nonlinear optical systems. The spectral bifurcation doesn't arise due to the gain saturation induced nonlinearity (see Supplementary Information, Section 9). This is also corroborated by the existence of the quantum image of this above-threshold phenomenon below threshold (where gain saturation is absent) (see Supplementary Information, Section 10) which is consistent with the theoretical predictions in the spatial domain ${ }^{48}$.

In summary, we have performed complete characterization of this second-order phase transition both in the temporal and in the spectral (optical and radio-frequency) domain. Experimental results are backed by numerical simulations of the underlying spatio-temporal phenomena and corroborated using mean-field 

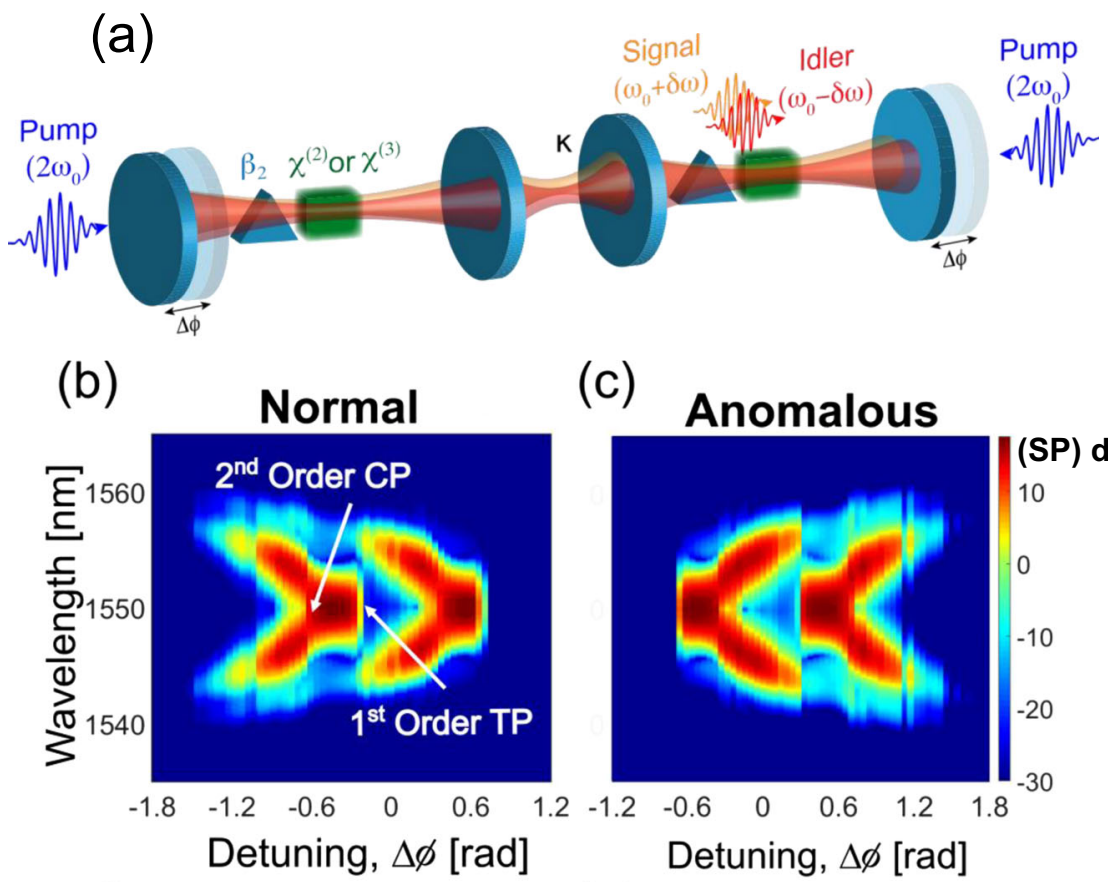

(c) Anomalous

(d)
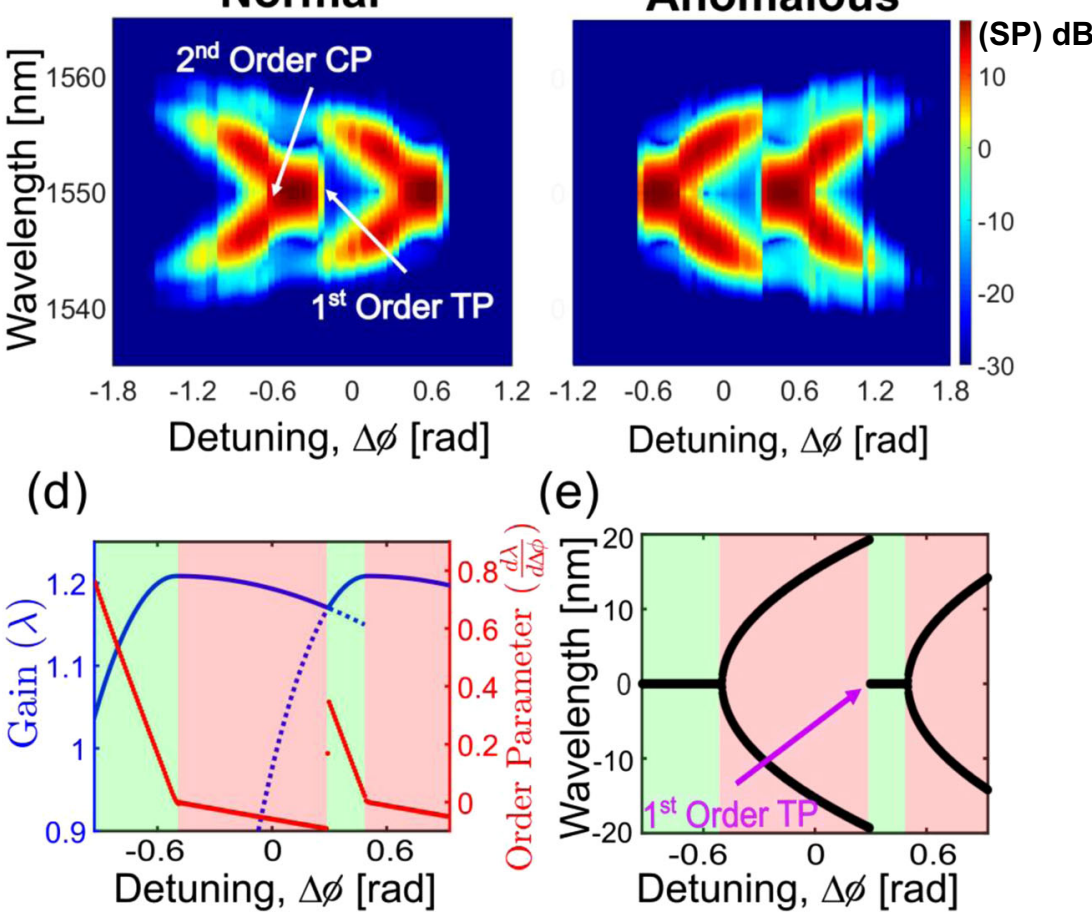

(e)

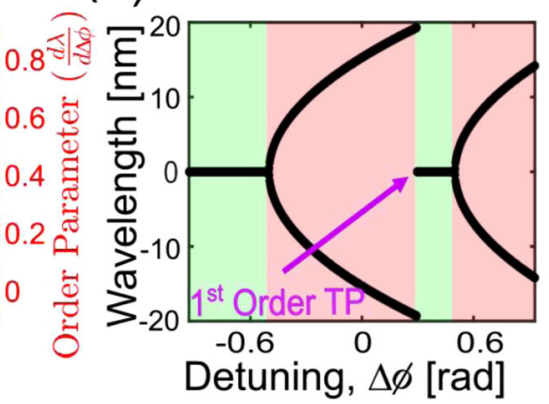

Fig. 4 First-order spectral phase transition in coupled OPOs. a Schematic configuration of a coupled OPO (coupling factor ( $\kappa$ )). Simulated spectrum (SP) as a function of the cavity detuning $\mathbf{b}$ in the normal dispersion regime, $\mathbf{c}$ in the anomalous dispersion regime. $\mathbf{d}$ Order parameter as a function of detuning showing the discontinuity at the location of the first-order transition point. The gain curve is also plotted alongside. The OPO selects the gain maximum and therefore doesn't follow the dashed portion of the gain curve. This gain competition between two second-order critical point gives rise to the first-order transition point. e The spectrum considering the wavelength experiencing the maximum gain around which the signal/idler is centered. At the first-order transition, there is a discontinuous jump from the non-degenerate spectrum to the degenerate spectrum.

analytical descriptions. We have shown that some of the nonequilibrium spectral behaviors of OPOs can be formulated as a universal phase transition.

The semi-classical spectral phase transition considered in this work can be extended to the quantum regime below threshold opening a path toward a quantum phase transition in the spectral domain. The ability to engineer the dispersion of integrated $\chi^{(2)}$ and $\chi^{(3)}$ devices ${ }^{49}$ will allow manipulation of the spectral phase transition behavior. Probing the performance difference of sensors based on second-order phase transitions and those leveraging second-order exceptional points ${ }^{28,29}$ is a subject of future work.

\section{Methods}

Experimental setup. The experimental schematic is shown in Fig. 2a, a detailed version of which is presented as Fig. S1 (Supplementary Information, Section 2). The OPO pump is derived from the mode-locked laser through second harmonic generation (SHG) in a quasi-phase matched periodically poled lithium niobate (PPLN) crystal. The pump is centered around $775 \mathrm{~nm}$. The main cavity is composed of a PPLN waveguide (reverse proton exchange, $40 \mathrm{~mm}$ long, periodically poled to phase-match $775-1550 \mathrm{~nm}$ interaction $)^{50}$ with fiber coupled output ports, fiber phase shifter, free-space section (to adjust the pump repetition rate to be multiple of the free spectral range of the cavity.), additional fiber segment to engineer the cavity dispersion, and a beam splitter which provides the output coupling. All fibers and devices existing in the optical path are single mode, polarization maintaining and connectors are angle polished. Additional details pertaining to the experimental setup/methods are provided in the supplementary information (Supplementary Information, Section 2).

System modeling. The nonlinear interaction inside the PPLN waveguide is governed by:

$$
\begin{gathered}
\frac{\partial a}{\partial z}=\left[-\frac{\alpha^{(a)}}{2}-i \frac{\beta_{2}^{(a)}}{2 !} \frac{\partial^{2}}{\partial t^{2}}+\ldots\right] a+\epsilon a^{*} b \\
\frac{\partial b}{\partial z}=\left[-\frac{\alpha^{(b)}}{2}-u \frac{\partial}{\partial t}-i \frac{\beta_{2}^{(b)}}{2 !} \frac{\partial^{2}}{\partial t^{2}}+\ldots\right] b-\frac{\epsilon a^{2}}{2}
\end{gathered}
$$

The evolution of the $\operatorname{signal}(a)$ and the $\operatorname{pump}(b)$ envelopes in the slowly varying envelope approximation is dictated by Eqs. (4a) and (4b), respectively ${ }^{35}$. The effects of higher-order GVD $\beta_{2}, \beta_{3}$, group-velocity mismatch (GVM) $(u)$, the back-conversion from the signal to the pump are included. The round-trip feedback is given by:

$$
\begin{gathered}
a^{(n+1)}(0, t)=\mathcal{F}^{-1}\left\{G_{0}^{-\frac{1}{2}} e^{i \bar{\phi}} \mathcal{F}\left\{a^{(n)}(L, t)\right\}\right\} \\
\bar{\phi}=\Delta \phi+\frac{l \lambda^{(a)}}{2 c}\left(\omega-\omega_{0}\right)+\frac{\phi_{2}}{2 !}\left(\omega-\omega_{0}\right)^{2}+\ldots
\end{gathered}
$$

Equation (5b) takes into consideration the round-trip loss which is lumped into an aggregated out-coupling loss factor $G_{0}$, the GVD $\left(\phi_{2}\right)$ of the feedback path and 
(a)

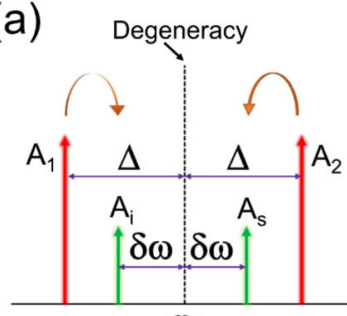

$\omega 0$

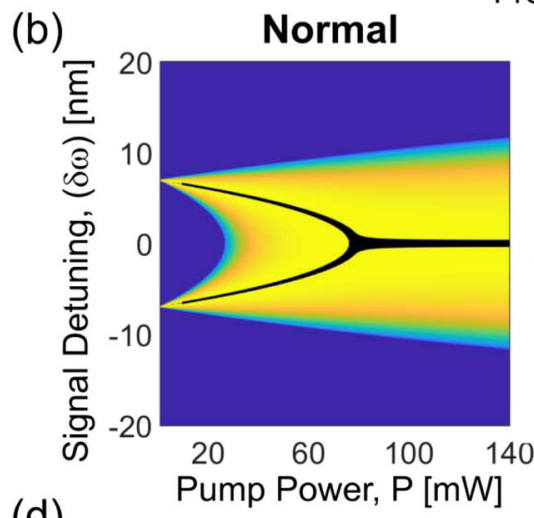

.

(c)

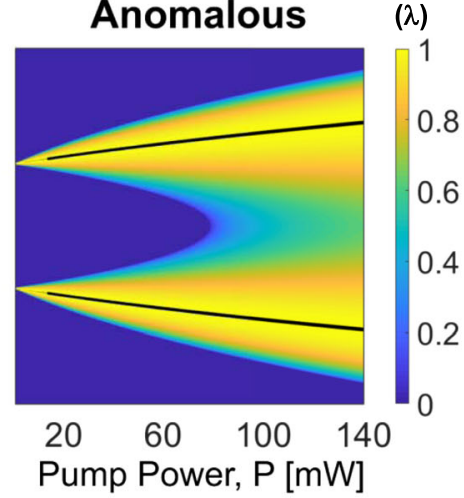

$(\lambda)$

0.6

0.4

(e)

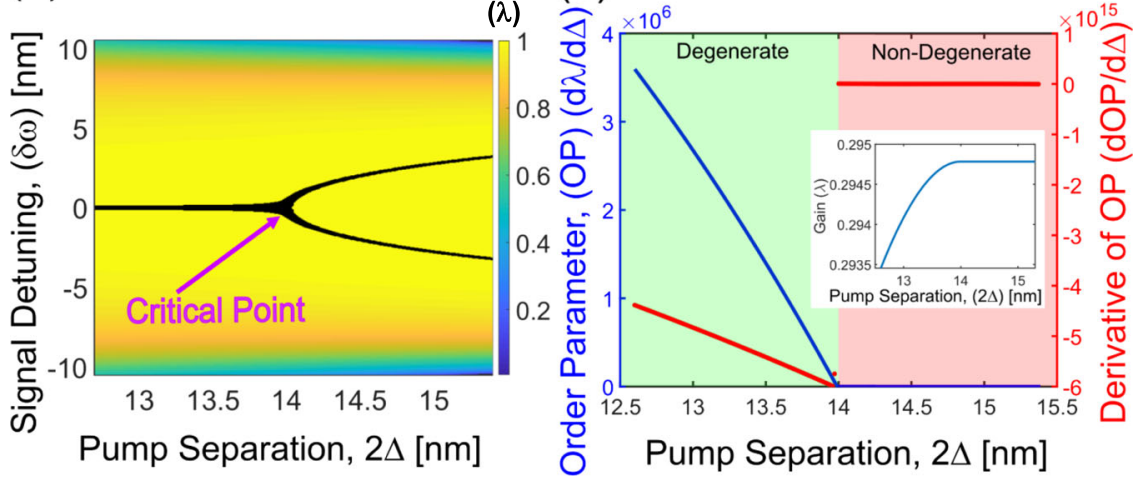

Fig. 5 Spectral phase transition in dual-pump four-wave mixing. a Illustration of the two pump fields getting converted to the signal and idler. $\mathbf{b}$ The phase conjugation parametric instability gain ( $\lambda$ ) (Eq. (3)) curve in the normal dispersion regime as the pump power is varied. The maximum of the gain where the signal/idler is supposed to oscillate is marked by the black lines. Degenerate and non-degenerate oscillations are both expected in this case. c The phase conjugation parametric process gain curve in the anomalous dispersion regime. Only non-degenerate oscillation is expected in this case. $\mathbf{d}$ Spectral phase transition (normal dispersion regime) as the pump separation $(\Delta)$ is varied. A degenerate to non-degenerate transition happens across the critical point. e The critical point is characterized to be a second-order which displays continuous behavior in order parameter but exhibits discontinuity in its derivative. Parameters used in the simulation are taken from ref. ${ }^{42}$.

the detuning $(\Delta \phi)\left(\Delta \phi=\pi l+\phi_{0}, l\right.$ is the cavity length detuning in units of signal half-wavelengths in vacuum) of the circulating signal from the exact synchrony with respect to the pump. The effective second-order nonlinearity co-efficient $(\epsilon)$ is related to the SHG efficiency ${ }^{35}$. The round-trip number is denoted by $n$ and the cavity length by $L$. The equations are numerically solved adopting the split-step Fourier algorithm.

To explain the spectral phase transition phenomenon numerically and analytically we adopt a two-pronged approach. First, we develop a mean-field model in the high finesse, CW driven limit and provide an analytical description for the occurrence of the spectral phase transition. This model, though does not inherit all the characteristics of the synchronously pumped optical parametric oscillator, is able to encapsulate the qualitative nature of the spectral phase transition. Secondly, we calculate the eigenvalue which is related to the gain of the signal/idler per roundtrip by assuming the pump in the OPO to be effectively CW having an average power being equal to the peak power of the pump pulse (see Supplemental Information, Section 4).

For Kerr OPO, the evolution of the optical fields in the non-resonant Kerr nonlinear medium is governed by the nonlinear Schrodinger equation. The larger eigenvalue $(\lambda)$ of the linear stability matrix of the phase conjugation nonlinear interaction determines the gain and is obtained in the undepleted pump approximation (see Supplementary Information, Section 14). However, in a Kerr nonlinear medium additional nonlinear interactions, namely the modulation instability and four-wave mixing Bragg scattering accompany the phase conjugation process responsible for the phase-sensitive degenerate parametric oscillation. Spectral phase transitions can also be investigated in a drivendissipative setting in a Kerr resonator, with the detuning between the pumps and the cold-cavity resonances being an additional degree of freedom.

Reporting summary. Further information on research design is available in the Nature Research Reporting Summary linked to this article.

\section{Data availability}

The data that support the plots within this paper and other findings of this study are available from the corresponding author.

\section{Code availability}

The codes that support the findings of this study are available from the corresponding author upon reasonable request. 
Received: 2 April 2020; Accepted: 11 January 2021;

Published online: 05 February 2021

\section{References}

1. Vahala, K. J. Optical microcavities. Nature 424, 839 (2003).

2. Limonov, M. F., Rybin, M. V., Poddubny, A. N. \& Kivshar, Y. S. Fano resonances in photonics. Nat. Photonics 11, 543 (2017).

3. Özdemir, Ş., Rotter, S., Nori, F. \& Yang, L. Parity-time symmetry and exceptional points in photonics. Nat. Mater. 18, 783 (2019).

4. Miri, M.-A., \& Alu, A. Exceptional points in optics and photonics. Science 363 , $42(2019)$.

5. Ropp, C., Bachelard, N., Barth, D., Wang, Y. \& Zhang, X. Dissipative selforganization in optical space. Nat. Photonics 12, 739 (2018).

6. Vaupel, M., Maitre, A. \& Fabre, C. Observation of pattern formation in optical parametric oscillators. Phys. Rev. Lett. 83, 5278 (1999).

7. Bortolozzo, U., Villoresi, P. \& Ramazza, P. Experimental evidence for detuning induced pattern selection in nonlinear optics. Phys. Rev. Lett. 87, 274102 (2001).

8. Lugiato, L. A. \& Lefever, R. Spatial dissipative structures in passive optical systems. Phys. Rev. Lett. 58, 2209 (1987)

9. Taranenko, V., Staliunas, K. \& Weiss, C. Pattern formation and localized structures in degenerate optical parametric mixing. Phys. Rev. Lett. 81, 2236 (1998).

10. Oppo, G.-L., Yao, A. M. \& Cuozzo, D. Self-organization, pattern formation, cavity solitons, and rogue waves in singly resonant optical parametric oscillators. Phys. Rev. A 88, 043813 (2013).

11. Cross, M. C. \& Hohenberg, P. C. Pattern formation outside of equilibrium. Rev. Mod. Phys. 65, 851 (1993).

12. Kuznetsov, A. Optical bistability driven by a first order phase transition. Opt. Commun. 81, 106 (1991).

13. Marowsky, G. \& Heudorfer, W. Second and first order phase transition analogy in the operation of an organic dye laser. Opt. Commun. 26, 381 (1978).

14. Haken, H. Cooperative phenomena in systems far from thermal equilibrium and in nonphysical systems. Rev. Mod. Phys. 47, 67 (1975).

15. DeGiorgio, V. \& Scully, M. O. Analogy between the laser threshold region and a second-order phase transition. Phys. Rev. A 2, 1170 (1970).

16. Leonetti, M., Conti, C. \& Lopez, C. The mode-locking transition of random lasers. Nat. Photonics 5, 615 (2011).

17. Gordon, A. \& Fischer, B. Phase transition theory of many-mode ordering and pulse formation in lasers. Phys. Rev. Lett. 89, 103901 (2002).

18. Fischer, B. \& Bekker, A. Many-body photonics. Opt. Photonics N. 24, 40 (2013).

19. Herr, T. et al. Temporal solitons in optical microresonators. Nat. Photonics $\mathbf{8}$, 145 (2014).

20. Carusotto, I. \& Ciuti, C. Quantum fluids of light. Rev. Mod. Phys. 85, 299 (2013).

21. Bodenschatz, E., Pesch, W. \& Ahlers, G. Recent developments in rayleighbénard convection. Annu. Rev. fluid Mech. 32, 709 (2000).

22. Engels, P., Atherton, C. \& Hoefer, M. Observation of faraday waves in a boseeinstein condensate. Phys. Rev. Lett. 98, 095301 (2007).

23. Drummond, P., McNeil, K. \& Walls, D. Non-equilibrium transitions in sub/ second harmonic generation. Opt. Acta.: Int. J. Opt. 27, 321 (1980).

24. Dunnett, K. \& Szymańska, M. Keldysh field theory for nonequilibrium condensation in a parametrically pumped polariton system. Phys. Rev. B 93, 195306 (2016).

25. Dagvadorj, G. et al. Nonequilibrium phase transition in a two-dimensional driven open quantum system. Phys. Rev. X 5, 041028 (2015).

26. Stanley, H. E. Phase Transitions and Critical Phenomena (Clarendon Press, Oxford, 1971).

27. Gol'Tsman, G. et al. Picosecond superconducting single-photon optical detector. Appl. Phys. Lett. 79, 705 (2001)

28. Hodaei, H. et al. Enhanced sensitivity at higher-order exceptional points. Nature 548, 187 (2017).

29. Lai, Y.-H., Lu, Y.-K., Suh, M.-G., Yuan, Z. \& Vahala, K. Observation of the exceptional-point-enhanced Sagnac effect. Nature 576, 65-69 (2019).

30. Wang, H., Lai, Y.-H., Yuan, Z., Suh, M.-G. \& Vahala, K. Petermann-factor sensitivity limit near an exceptional point in a Brillouin ring laser gyroscope. Nature communications 11, 1-6 (2020).

31. Lau, H.-K. \& Clerk, A. A. Fundamental limits and non-reciprocal approaches in non-hermitian quantum sensing. Nat. Commun. 9, 4320 (2018).

32. Caves, C. M. Quantum limits on noise in linear amplifiers. Phys. Rev. D. 26, 1817 (1982).
33. De Valcarcel, G., Staliunas, K., Roldán, E. \& Sánchez-Morcillo, V. Transverse patterns in degenerate optical parametric oscillation and degenerate four-wave mixing. Phys. Rev. A 54, 1609 (1996).

34. Longhi, S. \& Geraci, A. Swift-hohenberg equation for optical parametric oscillators. Phys. Rev. A 54, 4581 (1996).

35. Hamerly, R. et al. Reduced models and design principles for half-harmonic generation in synchronously pumped optical parametric oscillators. Phys. Rev. A 94, 063809 (2016).

36. Trillo, S. \& Haelterman, M. Parametric solitons in passive structures with feedback. In Spatial Solitons, 359-393 (Springer, 2001).

37. Marandi, A., Wang, Z., Takata, K., Byer, R. L. \& Yamamoto, Y. Network of time-multiplexed optical parametric oscillators as a coherent ising machine. Nat. Photonics 8, 937 (2014).

38. Coen, S., Randle, H. G., Sylvestre, T. \& Erkintalo, M. Modeling of octavespanning kerr frequency combs using a generalized mean-field lugiato-lefever model. Opt. Lett. 38, 37 (2013).

39. Nabors, C., Yang, S., Day, T. \& Byer, R. Coherence properties of a doubly resonant monolithic optical parametric oscillator. JOSA B 7, 815 (1990).

40. Fabre, C., Giacobino, E., Heidmann, A. \& Reynaud, S. Noise characteristics of a non-degenerate optical parametric oscillator-application to quantum noise reduction. J. de. Phys. 50, 1209 (1989).

41. Strogatz, S. H. Nonlinear Dynamics and Chaos: with Applications to Physics, Biology, Chemistry, and Engineering (CRC Press, 2018).

42. Okawachi, Y. et al. Dual-pumped degenerate kerr oscillator in a silicon nitride microresonator. Opt. Lett. 40, 5267 (2015)

43. Inagaki, T. et al. Large-scale ising spin network based on degenerate optical parametric oscillators. Nat. Photonics 10, 415 (2016).

44. Yang, L.-P. \& Jacob, Z. Quantum critical detector: amplifying weak signals using discontinuous quantum phase transitions. Opt. Express 27, 10482 (2019).

45. Hamerly, R. et al. Experimental investigation of performance differences between coherent ising machines and a quantum annealer. Sci. Adv. $\mathbf{5}$, eaau0823 (2019).

46. Kalinin, K. P., Amo, A., Bloch, J. \& Berloff, N. G. Polaritonic XY-Ising machine. Nanophotonics, 9, 4127-4138 (2020).

47. Eckardt, R. C., Nabors, C., Kozlovsky, W. J. \& Byer, R. L. Optical parametric oscillator frequency tuning and control. JOSA B 8, 646 (1991).

48. Gatti, A. \& Lugiato, L. Quantum images and critical fluctuations in the optical parametric oscillator below threshold. Phys. Rev. A 52, 1675 (1995).

49. Wang, C., Zhang, M., Stern, B., Lipson, M. \& Lončar, M. Nanophotonic lithium niobate electro-optic modulators. Opt. express 26, 1547 (2018).

50. Langrock, C. \& Fejer, M. Fiber-feedback continuous-wave and synchronouslypumped singly-resonant ring optical parametric oscillators using reverseproton-exchanged periodically-poled lithium niobate waveguides. Opt. Lett. 32, 2263 (2007).

\section{Acknowledgements}

We acknowledge stimulating discussions with Avik Dutt, Mohammad-Ali Miri, Myoung-Gyun Suh, Li-Ping Yang, and Marc Jankowski. The authors gratefully acknowledge support from ARO grant no. W911NF-18-1-0285 and NSF grant no. 1846273 and 1918549, and AFOSR award FA9550-20-1-0040. The authors wish to thank NTT Research for their financial and technical support.

\section{Author contributions}

A.R. and A.M. conceived the idea and performed the experiments. A.R. and S.J. developed the theory and performed the numerical simulations. C.L. fabricated the PPLN waveguide used in the experiment with supervision of M.F. A.R. and A.M. wrote the manuscript with input from all authors. A.M. supervised the project.

\section{Competing interests}

The authors declare no competing interests.

\section{Additional information}

Supplementary information The online version contains supplementary material available at https://doi.org/10.1038/s41467-021-21048-z.

Correspondence and requests for materials should be addressed to A.M.

Peer review information Nature Communications thanks Kaled Dechoum and the other, anonymous, reviewer(s) for their contribution to the peer review of this work.

Reprints and permission information is available at http://www.nature.com/reprints

Publisher's note Springer Nature remains neutral with regard to jurisdictional claims in published maps and institutional affiliations. 
(c) (i) Open Access This article is licensed under a Creative Commons Attribution 4.0 International License, which permits use, sharing, adaptation, distribution and reproduction in any medium or format, as long as you give appropriate credit to the original author(s) and the source, provide a link to the Creative Commons license, and indicate if changes were made. The images or other third party material in this article are included in the article's Creative Commons license, unless indicated otherwise in a credit line to the material. If material is not included in the article's Creative Commons license and your intended use is not permitted by statutory regulation or exceeds the permitted use, you will need to obtain permission directly from the copyright holder. To view a copy of this license, visit http://creativecommons.org/ licenses/by/4.0/.

(C) The Author(s) 2021 